\title{
Development of an Autonomous Two-Wheeled Vehicle Robot
}

\author{
Minoru Sasaki ${ }^{1, a}$, Hidenobu Tanaka ${ }^{1, b}$ and Satoshi Ito ${ }^{1, c}$ \\ ${ }^{1}$ Department of Human and Information Systems Engineering, Gifu University, \\ 1-1 Yanagido, Gifu, 501-1193, Japan \\ asasaki@gifu-u.ac.jp, bo3128017@edu.gifu-u.ac.jp, csatoshi@gifu-u.ac.jp
}

Keywords: two-wheeled vehicle robot, steering control, modeling, LQI control, system development

\begin{abstract}
This paper describes a development of an autonomous two-wheeled vehicle robot. The model of the two-wheeled vehicle using steering control is derived. The control systems are designed by linear quadratic regulator and linear quadratic integral method. Stabilization is achieved by measuring roll angle and roll rate and controlling the steering torque. The experimental results and simulation results show stable running control of the two-wheeled vehicle robot and coincident with each other. The approach is validated through these results.
\end{abstract}

\section{Introduction}

Unmanned motorcycles or two-wheeled vehicles are inherently unstable nonlinear systems. Those properties strongly depend on travel velocity and friction between tires and load surface which makes the design of a stabilizing controller for such unmanned vehicles particularly challenging. Ruijs and Pacejka were probably the first to stabilize a motorcycle by feedback control. Getz and Marsden developed a controller that used both steering angle and forward speed to control riderless bicycles. Chidzonga and Eitelberg investigated the possibility of stabilizing a bicycle at rest by controlling the velocity when the front wheel is steered at a fixed angle. Miyagishi et al. used a proportionalderivative controller to control the roll angle of an unmanned motorcycle. Iuchi, Niki and Murakami presented MIMO two-degree-of-freedom control scheme, using both steering angle and center-of-gravity lateral shifting in order to keep a bicycle upright. Yi et al. presented a trajectory tracking and balancing control algorithm for an autonomous motorcycle. Weir and Sharp showed that motorcycle steering is achieved mainly through steering torque, while body-induced shift of the center of gravity is secondary. In this paper the controller design is based on Linear Quadratic Integrator controller. The approach is validated through simulations and experiments of straight running and curving running of the two-wheeled vehicle robot.
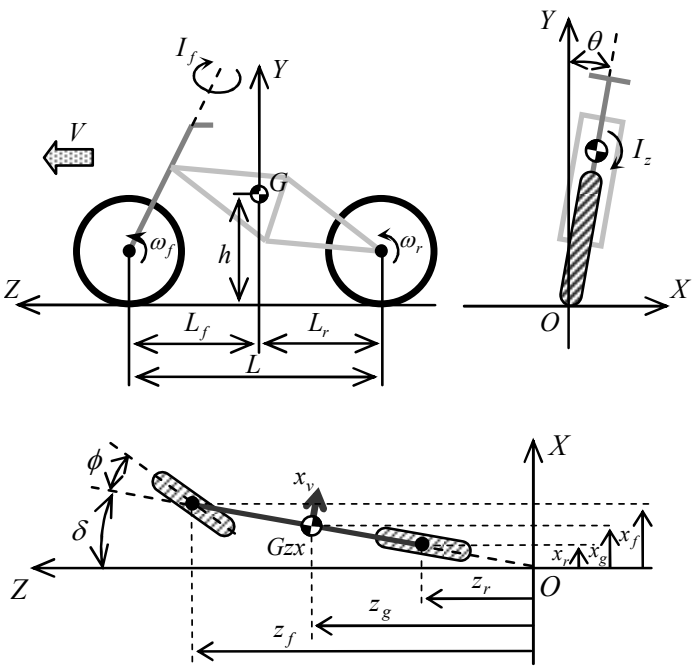

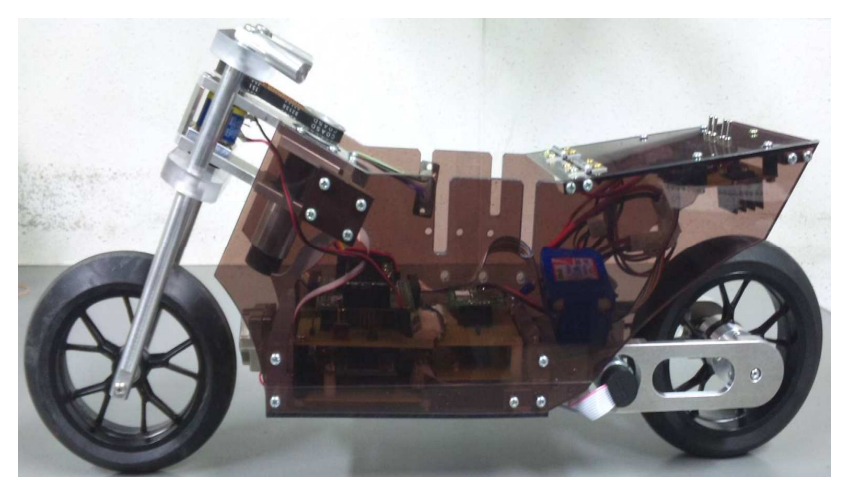

Fig. 1. Overview of the two-wheeled vehicle robot 

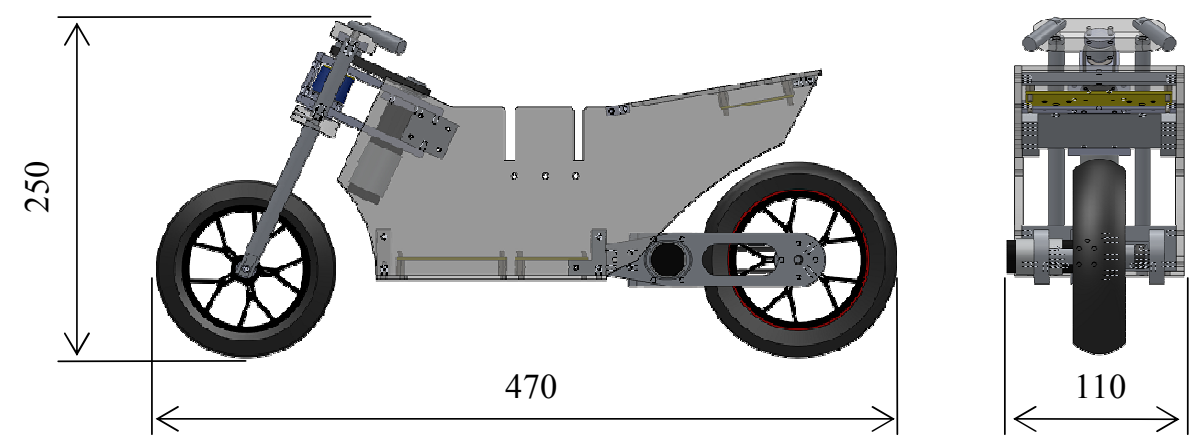

Fig. 2. Experimental prototype two-wheeled mobile robot

\section{Two-wheeled vehicle robot}

The experimental prototype robot with total length $470 \mathrm{~mm}$, hight $250 \mathrm{~mm}$, width $110 \mathrm{~mm}$, weight $2333 \mathrm{~g}$ and maximum speed $3.2 \mathrm{~m} / \mathrm{sec}$ is shown in Fig. 2. SH7144F microprocessor was installed on the robot and received roll angle, encoder outputs of steering angle and rear wheel rotation angle. Roll rate was measured using a vibrating ring gyroscope ( Silicon Sensing Systems, CRS03-02).

\section{Dynamic modeling}

In order to design the controller, a dynamic model of the two-wheeled robot was derived under the following assumptions.

1. The body rotational angle $\delta$ around $Y$ axis is small.

2. The steering angle $\phi$ and the tilt angle of the body $\theta$ are small.

3. Forward velocity is constant or changing slowly.

4. The center of gravity shifting of the robot is small when the steering angle changed.

5. The steering control is speed control system based on motor driver.

Under these assumptions, using the symbols defined as shown in Fig. 2, the following roll and steering equations are obtained;

$$
\begin{aligned}
& \left(I_{z}+m h^{2}\right) \ddot{\theta}=m g h \theta-\frac{V}{L}\left\{m h\left(L_{r}+L_{f}\right) \frac{V}{L}+I_{f} \omega_{f}+I_{r} \omega_{r}\right\} \phi-\left(\frac{V}{L} m h L_{r}+I_{f} \omega_{f}\right) \dot{\phi} \\
& \ddot{\phi}+\alpha \dot{\phi}+\beta \phi=\mu
\end{aligned}
$$

And linearized roll and steering state space equations, state vector and output equation are obtained:

$$
\begin{aligned}
& \dot{\boldsymbol{x}}(t)=\boldsymbol{A} \boldsymbol{x}(t)+\boldsymbol{B} \boldsymbol{u}(t) \\
& \boldsymbol{y}(t)=\boldsymbol{C} \boldsymbol{x}(t)+\boldsymbol{D} \boldsymbol{u}(t)
\end{aligned}
$$

where

$$
\begin{aligned}
& \boldsymbol{A}=\left[\begin{array}{cccc}
0 & 0 & 1 & 0 \\
0 & 0 & 0 & 1 \\
a_{31} & a_{32} & a_{33} & a_{34} \\
a_{41} & a_{42} & a_{43} & a_{44}
\end{array}\right], \boldsymbol{B}=\left[\begin{array}{c}
0 \\
0 \\
b_{3} \\
b_{4}
\end{array}\right], \boldsymbol{C}=\left[\begin{array}{cccc}
1 & 0 & 0 & 0 \\
0 & 1 & 0 & 0 \\
0 & 0 & 1 & 0 \\
0 & 0 & 0 & 1
\end{array}\right], \boldsymbol{D}=\left[\begin{array}{c}
0 \\
0 \\
0 \\
0
\end{array}\right], \boldsymbol{x}(t)=\left[\begin{array}{c}
\theta \\
\phi \\
\dot{\theta} \\
\dot{\phi}
\end{array}\right], \\
& \left\{\begin{array}{l}
a=I_{z}+m h^{2} \\
b=m g h \\
c=\frac{V}{L}\left\{m h\left(L_{r}+L_{f}\right) \frac{V}{L}+I_{f} \omega_{f}+I_{r} \omega_{r}\right\}
\end{array}\right. \\
& d=\frac{V}{L} m h L_{r}+I_{f} \omega_{f} \\
& a_{31}=\frac{b}{a}, \quad a_{32}=\frac{c}{a}, \quad a_{33}=0, \quad a_{34}=\frac{d}{a}, \quad b_{3}=0 \\
& a_{41}=0, \quad a_{42}=-\beta, \quad a_{43}=0, \quad a_{44}=-\alpha, \quad b_{4}=\gamma
\end{aligned}
$$


Properties of the two-wheeled robot were estimated either through direct measurements, e.g. physical dimensions and centers of mass; or by Computer Aided Design (Solidworks ${ }^{\mathrm{TM}}$ ) models based on measurements, e.g. calculation of moments of inertia. The parameters of the steering part were experimentally identified as shown in Fig. 3. Very little plant uncertainty was observed.

\section{Controller design}

In several control designs there is a wealth of experience and knowledge that dictates in many situations what sort of compensator dynamics yield good performance from the point of view of the controls engineer. For example, a washout circuit may be required, or it may be necessary to augment some feed forward channels with integrators to obtain a steady-state error of exactly zero. The control system structures used in classical aircraft design also give good robustness properties. That is, they perform well even if there are disturbances or uncertainties in the system. Our approach to tracker design allows controller dynamics of any desired structure, and then determines the control gains that minimize a quadratic PI over that structure. A dynamic compensator of prescribed structure may be incorporated into the system description as follows. Consider a state variable model of the plant given by Equations (3) and (4). We begin by considering a familiar design problem, the design of a controller to enable the tracking of a step reference input with zero steady-state error. The tracking error $e$ is defined as

$$
e=y-r
$$

Taking the time derivative yields

$$
\frac{d}{d t} e(t)=C \dot{x}(t)
$$
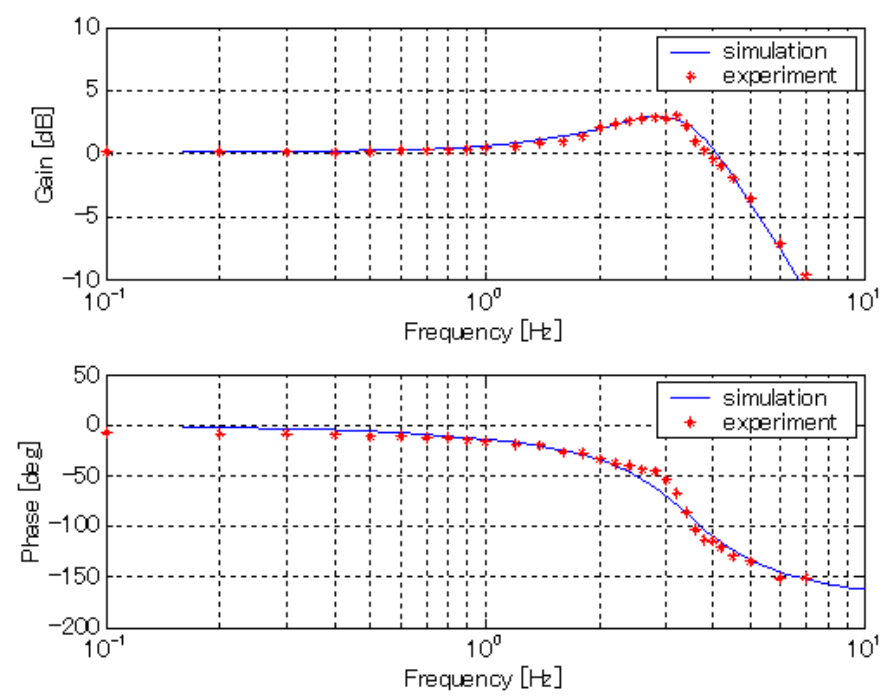

Fig. 3. Frequency response of the steering part

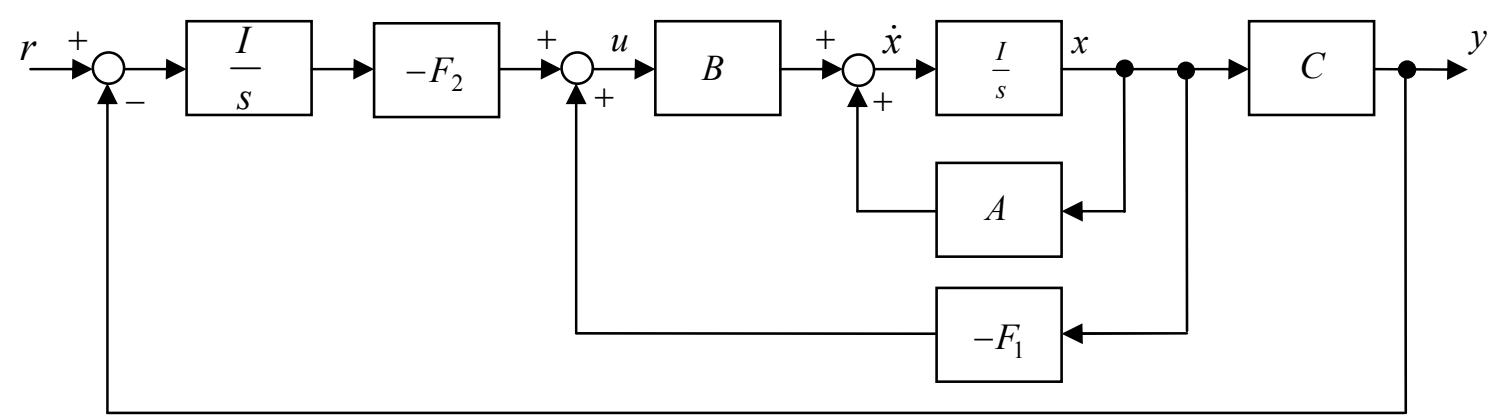

Fig. 4. Block Diagram of LQI 
These dynamics and output equations may be written in augmented form as

$$
\frac{d}{d t}\left[\begin{array}{l}
\dot{x}(t) \\
e(t)
\end{array}\right]=\left[\begin{array}{ll}
A & 0 \\
C & 0
\end{array}\right]\left[\begin{array}{l}
\dot{x}(t) \\
e(t)
\end{array}\right]+\left[\begin{array}{l}
B \\
0
\end{array}\right] \dot{u}(t)
$$

If the system is controllable, we find a feedback of the form

$$
\dot{u}(t)=-F_{1} \dot{x}(t)-F_{2} e(t)
$$

such that Eq. (7) is stable. This implies that the tracking error $e$ is stable; thus we will have achieved the objective of asymptotic tracking with zero steady-state error. The control input, found by integrating Eq. (8), is

$$
u(t)=-F_{1} x(t)-F_{2} \int_{0}^{t} e(t) d t
$$

The corresponding block diagram is shown in Fig. 5. We see that the compensator includes an internal model of the reference step input.

\begin{tabular}{ccc}
\multicolumn{3}{c}{ Table 1 Nominal value of the system } \\
\hline Nomenctature & Value & Unit \\
\hline$m$ & 2.33 & {$[\mathrm{~kg}]$} \\
$h$ & 0.096 & {$[\mathrm{~m}]$} \\
$L$ & 0.360 & {$[\mathrm{~m}]$} \\
$L_{f}$ & 0.195 & {$[\mathrm{~m}]$} \\
$L_{r}$ & 0.165 & {$[\mathrm{~m}]$} \\
$R_{f}$ & 0.058 & {$[\mathrm{~m}]$} \\
$R_{r}$ & 0.064 & {$[\mathrm{~m}]$} \\
$I_{z}$ & $7.35 \times 10^{-3}$ & {$\left[\mathrm{kgm}^{2}\right]$} \\
$I_{h}$ & $2.45 \times 10^{-4}$ & {$\left[\mathrm{kgm}^{2}\right]$} \\
$I_{f}$ & $2.51 \times 10^{-4}$ & {$\left[\mathrm{kgm}^{2}\right]$} \\
$I_{r}$ & $5.32 \times 10^{-4}$ & {$\left[\mathrm{kgm}^{2}\right]$} \\
$k_{m}$ & 0.017 & {$[\mathrm{Nm} / \mathrm{A}]$} \\
$R_{m}$ & 9.72 & {$[\Omega]$} \\
$n$ & 19 & {$[-]$} \\
$g$ & 9.8 & {$\left[\mathrm{~m} / \mathrm{s}^{2}\right]$} \\
$\alpha$ & 17 & {$[-]$} \\
$\beta$ & -5 & {$[-]$} \\
$\gamma$ & 19 & {$[-]$} \\
\hline
\end{tabular}

\section{Results and discussion}

Straight running results. After repeating the design using several different $R$ and $Q$, the weighting parameters are decided on $R=5, Q=\operatorname{diag}\left[\begin{array}{lllll}3000 & 1 & 10 & 1 & 20000\end{array}\right]$. With this $R$ and $Q$ the control matrix was $K_{1}=\left[\begin{array}{llll}117.1678 & -28.1724 & 10.5698 & -2.5429\end{array}\right], K_{2}=[141.414]$.

Figure 5 shows the results of simulations and experiments based on the designed controller with the sampling time $0.01[\mathrm{sec}]$, the running speed $1.5[\mathrm{~m} / \mathrm{sec}]$ and the reference body roll angle and steering angle are zero. It can be seen that the controller stabilizes the robot close to the vertical position. The good agreement between simulated and actual results is evident.

Curving running results. Figure 6 shows the results of simulations and experiments of the curving behavior of the robot with same conditions of straight running case. After $3 \mathrm{sec} 10$ [deg] step of the desired roll angle was changed. During all these tests the robot remained stable. Small fluctuations, in the range of $-0.008436-+0.008436[\mathrm{rad} / \mathrm{sec}]$ about the zero roll rate angle, are most probably due to the fact that the sensor sets the dead zone for the drift of the sensor. However, simulated results and experimental results are almost agreement each other. 


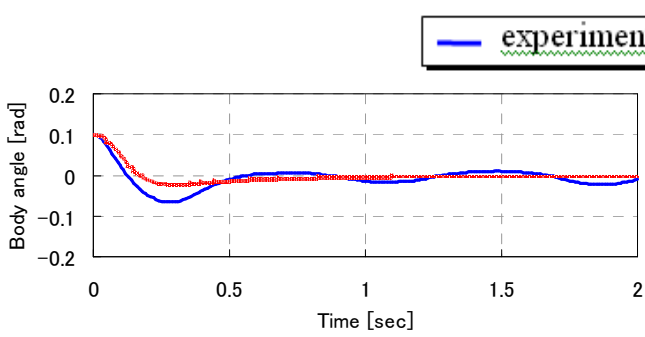

(a) Angle of body

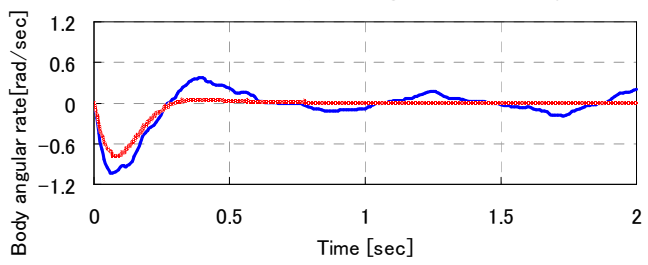

(c) Angular rate of body



(b) Angle of steering

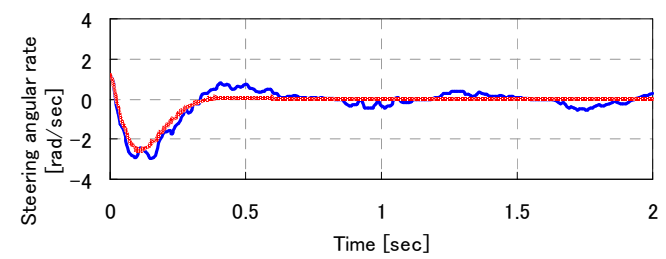

(d) Angular rate of steering

Fig. 5. Comparison of simulation and experiment results of straight running of the robot

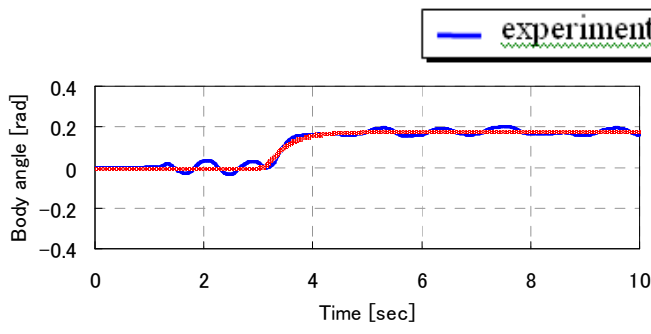

(a) Angle of body

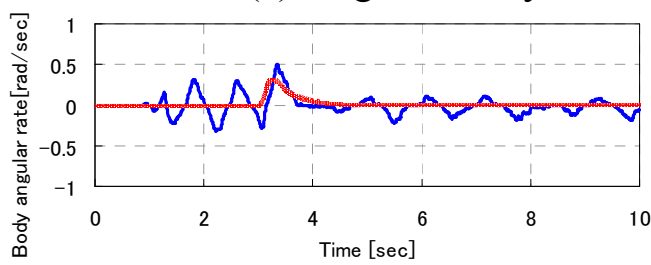

(c) Angular rate of body

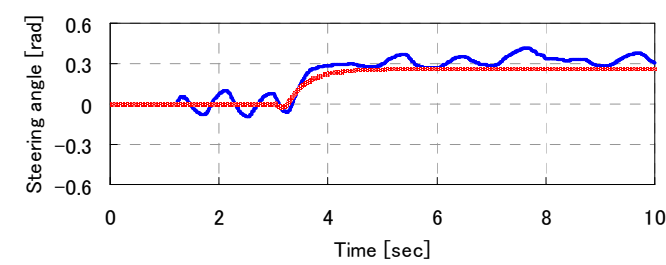

(b) Angle of steering

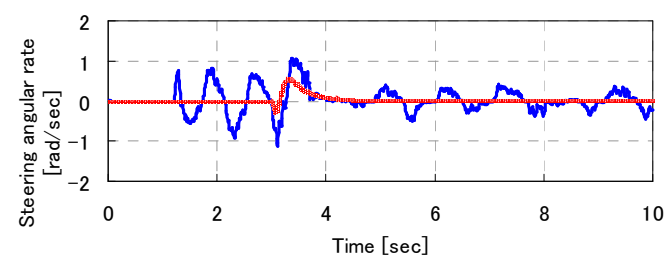

(d) Angular rate of steering

Fig. 6. Comparison of simulation and experiment results of curving behavior of the robot

\section{Conclusions}

A LQI controller that stabilizes an unmanned two-wheeled vehicle robot was developed and validated this controller was implemented on an experimental two-wheeled vehicle robot that was successfully autonomously operated on an carpet surface.

\section{References}

[1] Beznos, A.V.,Formal'sky,A.M.,Gurfinkel,E.V.,Jicharev,D.N.,Lensky,A.V.,Savitsky, K.V.,etal. (1998).Control of autonomous motion of two-wheel bicycle with gyroscopic stabilisation. In Proceedings of the 1998 IEEE international conference on robotics and automation.

[2] Chidzonga, R.F., \& Eitelberg, E.(2003).Controlling velocity and steering for bicycle stabilization. In Proceedings of the first African control conference. Available at /www.nt.ntnu.no/users/skoge/prost/proceedings/afcon03/Papers/010.pdf

[3] Getz, N.H.,\& Marsden, J.E.(1995). Control for an autonomous bicycle. In Proceedings of the 1995 IEEE international conference on robotics and automation. 
[4] Gutman, P.O. (1996). Qsyn. The toolbox for robust control systems design for use with MATLAB User's guide. Available at /http://www.math.kth.se/optsyst/forskning/forskarutbildning/5B5782/index.html.

[5] Gutman, P.-O.(2003). Robust and adaptive control. Fidelity or a free relation ship?. Systems and Control Letters 49, 9.19.

[6] Horowitz, I.M.(1993). Quantitative feedback design theory,.

[7] Horowitz, I.M., \& Sidi, M.(1972). Synthesis of feedback systems with large plant ignorance for prescribed time-domain tolerances. International Journal of Control, 16, 287.309.

[8] Iuchi, K., Niki, H., \& Murakami, T. (2005). Attitude control of bicycle motion by steering angle and variable COG control. In Proceedings of the $32^{\text {nd }}$ IEEE/IECON annual conference.

[9] Meijaard, J.P., Papadopoulos, J.M., Ruina, A., \& Schwab, A.L. (2007). Linearized dynamics equations for the balance and steer of a bicycle: A benchmark and review. Proceedings of the Royal Society A, 463, 1955.1982.

[10] Miyagishi, S. , Kageyama, I., Takama, K. ,Baba, M., \& Uchiyama, H. (2003). Study on construction of arider robot for two-wheeled vehicle. JSAE Review, 24, 321.326.

[11] Nenner, U.(2009). Control of an unmanned motor cycle robot. M. Sc. thesis, Technion Israel Institute of Technology.

[12] Nenner, U.,Linker, R., \& Gutman, P.O. (2009). Robust stabilization of an unmanned motor cycle. In Proceedings of the IEEE CIS-RAM conference, Chengdu, China.

[13] Ruijs, P.A.J., \& Pacejka, H.B. (1985). Recent research inlateral dynamics of motorcycles. Vehicle System Dynamics, 15, 467.480.

[14] Sharp, R.S. (1971). The stability and control of motor cycles. Journal of Mechanical Engineering Science, 13, 316.329.

[15] Sharp, R.S. (2001). Stability, control and steering response of motor cycles. Vehicle System Dynamics, 35, 291.318.

[16] Sharp, R.S. (2006). Optimal linear time-invariant preview steering control for motor cycles. Vehicle System Dynamics, 44, 329.340.

[17] Sharp, R.S. (2007a). Motor cycle steering control by road preview. Journal of Dynamic Systems, Measurements, and Control, 129, 373.381.

[18] Sharp, R.S.(2007b).Optimal preview speed-tracking control for motor cycles. Multi body Systems Dynamics, 18, 397.411.

[19] Sharp, R.S., Evangelou, S., \& Limebeer, D.J.N. (2004). Advances in the modeling of Motor cycle dynamics.. Multi body Systems Dynamics, 12, 251.283.

[20] Sharp, R.S., \& Limebeer, D.J.N. (2001). A motor cycle model for stability and control analysis. Multi body Systems Dynamics, 6, 123.142.

[21] Tanaka, Y., \& Murakami, T. (2004). Self sustaining bicycle robot with steering controller. In Proceedings of the eighth IEEE international workshop on advanced motion control.

[22] Weir, D.H. (1972). Motor cycle handling dynamics and rider control and the effect of design configurations on response and performance.Ph.D.thesis, University of California, LA.

[23] Yi, J., Song, D., Levandowski, A., \& Jayasuriya, S. (2006). Trajectory tracking and balance stabilization control of autonomous motor cycles. In Proceedings of the 2006 IEEE international conference on robotics and automation. 